\title{
Tracing intermediate phases during crystallization in a Ni-Zr metallic glass
}

Liu, S. Y.; Cao, Q. P.; Mu, X.; Xu, T. D.; Wang, D.; Ståhl, Kenny; Wang, X. D.; Zhang, D. X.; Kübel, C.; Jiang, J. Z.

Published in:

Acta Materialia

Link to article, DOI:

10.1016/j.actamat.2020.01.016

Publication date:

2020

Document Version

Peer reviewed version

Link back to DTU Orbit

Citation (APA):

Liu, S. Y., Cao, Q. P., Mu, X., Xu, T. D., Wang, D., Ståhl, K., Wang, X. D., Zhang, D. X., Kübel, C., \& Jiang, J. Z. (2020). Tracing intermediate phases during crystallization in a Ni-Zr metallic glass. Acta Materialia, 186, 396404. https://doi.org/10.1016/j.actamat.2020.01.016

\section{General rights}

Copyright and moral rights for the publications made accessible in the public portal are retained by the authors and/or other copyright owners and it is a condition of accessing publications that users recognise and abide by the legal requirements associated with these rights.

- Users may download and print one copy of any publication from the public portal for the purpose of private study or research.

- You may not further distribute the material or use it for any profit-making activity or commercial gain

- You may freely distribute the URL identifying the publication in the public portal 


\section{Journal Pre-proof}

Toxicity of dodecylbenzene to algae, crustacean, and fish - Passive dosing of highly hydrophobic liquids at the solubility limit

Felix Stibany, Stine Nørgaard Schmidt, Philipp Mayer, Andreas Schäffer

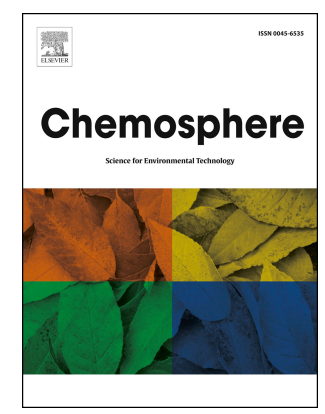

PII:

S0045-6535(20)30589-0

DOI:

https://doi.org/10.1016/j.chemosphere.2020.126396

Reference: $\quad$ CHEM 126396

To appear in: ECSN

Received Date: 7 January 2020

Revised Date: 28 February 2020

Accepted Date: 29 February 2020

Please cite this article as: Stibany, F., Schmidt, Stine.Nø., Mayer, P., Schäffer, A., Toxicity of dodecylbenzene to algae, crustacean, and fish - Passive dosing of highly hydrophobic liquids at the solubility limit, Chemosphere (2020), doi: https://doi.org/10.1016/j.chemosphere.2020.126396.

This is a PDF file of an article that has undergone enhancements after acceptance, such as the addition of a cover page and metadata, and formatting for readability, but it is not yet the definitive version of record. This version will undergo additional copyediting, typesetting and review before it is published in its final form, but we are providing this version to give early visibility of the article. Please note that, during the production process, errors may be discovered which could affect the content, and all legal disclaimers that apply to the journal pertain.

(C) 2020 Published by Elsevier Ltd. 
Toxicity of dodecylbenzene to algae, crustacean, and fish - Passive dosing of highly hydrophobic liquids at the solubility limit

Felix Stibany ${ }^{\mathrm{a}, \mathrm{b}, *}$, Stine Norgaard Schmidt ${ }^{\mathrm{b}}$, Philipp Mayer ${ }^{\mathrm{b}}$, and Andreas Schäffer ${ }^{\mathrm{a}}$

${ }^{\text {a } I n s t i t u t e ~ f o r ~ E n v i r o n m e n t a l ~ R e s e a r c h ~(B i o l o g y ~ V), ~ R W T H ~ A a c h e n ~ U n i v e r s i t y, ~}$ Worringerweg 1, 52074 Aachen, Germany

${ }^{\mathrm{b}}$ Department of Environmental Engineering, Technical University of Denmark, Bygningstorvet B115, 2800 Kgs. Lyngby, Denmark

* Corresponding author: felix.stibany@ rwth-aachen.de

\section{$\underline{\text { CRediT author statement }}$}

Felix Stibany: Conceptualization, Methodology, Validation, Formal analysis, Investigation, Writing - Original Draft, Writing - Review \& Editing, Visualization, Project administration

Stine Nørgaard Schmidt: Conceptualization, Methodology, Validation, Formal analysis, Writing - Original Draft, Writing - Review \& Editing

Philipp Mayer: Conceptualization, Methodology, Validation, Resources, Writing - Original Draft, Writing - Review \& Editing, Supervision, Project administration, Funding acquisition Andreas Schäffer: Conceptualization, Methodology, Validation, Resources, Writing Original Draft, Writing - Review \& Editing, Supervision, Project administration, Funding acquisition 
1 Toxicity of dodecylbenzene to algae, crustacean, and fish - Passive

2 dosing of highly hydrophobic liquids at the solubility limit

3

4 Felix Stibany $^{\mathrm{a}, \mathrm{b}, *}$, Stine Norgaard Schmidt ${ }^{\mathrm{b}}$, Philipp Mayer ${ }^{\mathrm{b}}$, and Andreas Schäffer ${ }^{\mathrm{a}}$

5

6 a Institute for Environmental Research (Biology V), RWTH Aachen University,

7 Worringerweg 1, 52074 Aachen, Germany

$8{ }^{\mathrm{b}}$ Department of Environmental Engineering, Technical University of Denmark,

9 Bygningstorvet B115, 2800 Kgs. Lyngby, Denmark

10

* Corresponding author: felix.stibany@ rwth-aachen.de

13 Current affiliation for Stine Nørgaard Schmidt: National Research Council, U.S.

14 Environmental Protection Agency, Office of Research and Development, Center for

15 Environmental Measurement and Modeling, Atlantic Coastal Environmental Sciences

16 Division, Narragansett, Rhode Island, USA 


\section{Graphical abstract}

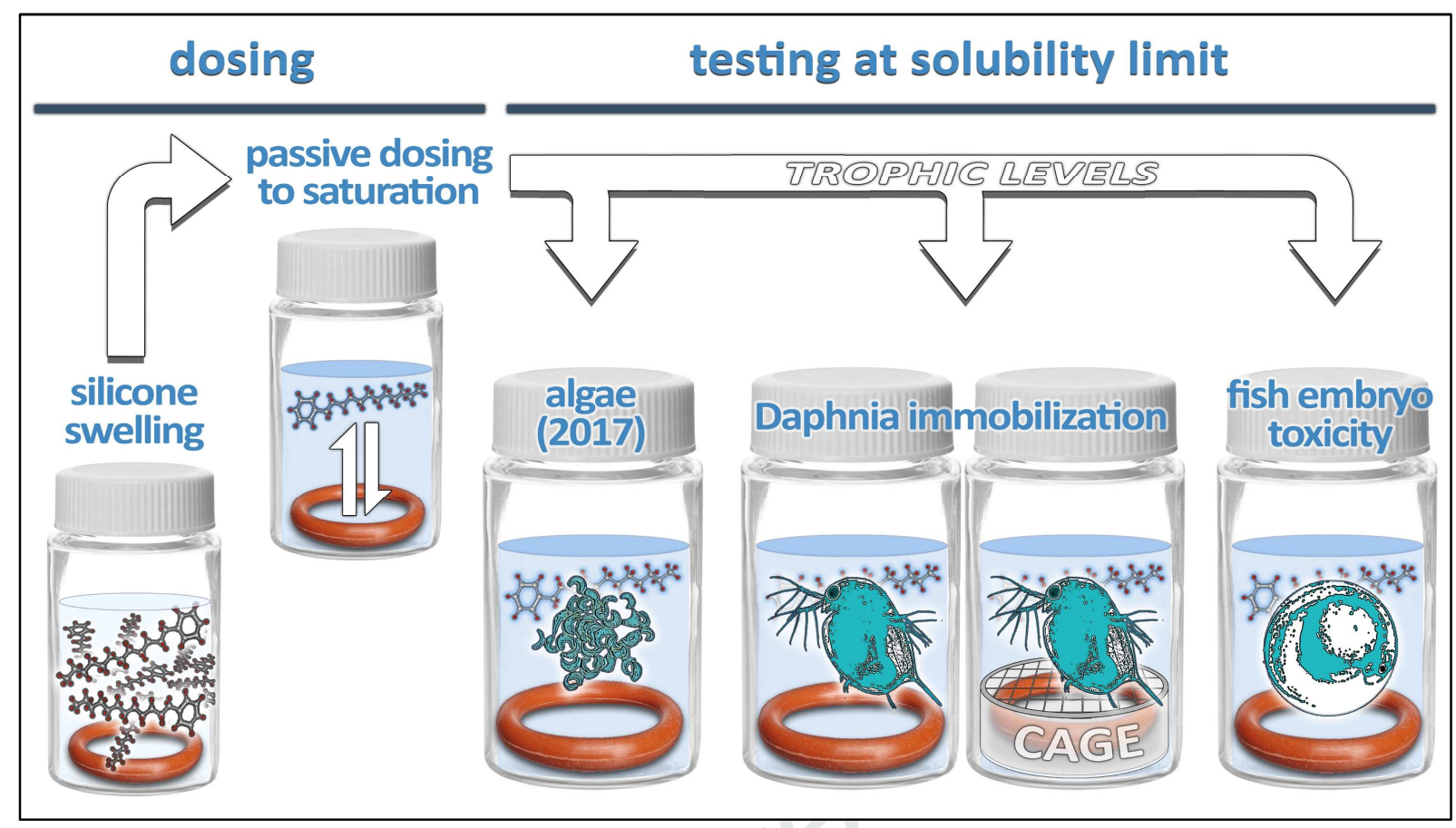

19

20 Keywords

21 Dodecylbenzene - highly hydrophobic - passive dosing - solubility limit - baseline toxicity -

22 chemical activity 


\section{Abstract}

In the current study, improved exposure control and measurements were applied for the aquatic toxicity testing of a highly hydrophobic organic compound. The aim was to reliably determine the ecotoxicity of the model compound dodecylbenzene (DDB, $\left.\log \mathrm{K}_{\mathrm{OW}}=8.65\right)$ by applying passive dosing for aquatic toxicity testing exactly at the solubility limit. Methodologically, silicone O-rings were saturated by immersion in pure liquid DDB (i.e., "loading by swelling") and then used as passive dosing donors. Daphnia immobilization and fish embryo toxicity tests were successfully conducted and provide, together with recently reported algal growth inhibition data, a full base-set of ecotoxicological data according to REACH. All tests were conducted in closed test systems to avoid evaporative losses, and exposure concentrations were measured throughout test durations. The Daphnia test was optimized by placing the O-rings in cages to prevent direct contact between daphnids and the passive dosing donor. Toxicologically, Daphnia magna immobilization was $19.3 \pm 8 \%$ (mean $\pm 95 \%$ CI; 6 tests) within 72 h, whereas Danio rerio fish embryos did not show any significant lethal or sublethal toxic responses within $96 \mathrm{~h}$. Growth rate inhibition for the algae Raphidocelis subcapitata was previously reported to be $13 \pm 5 \%$ in a first and $8 \pm 3 \%$ in a repeated test. These results for aquatic organisms, spanning three trophic levels, demonstrate toxicity of a highly hydrophobic compound and suggest that improvements of the current ecotoxicological standard tests are needed for these "difficult-to-test" chemicals. Furthermore, the obtained toxicity results significantly question the existence of a generic Log $\mathrm{K}_{\mathrm{OW}}$ cut-off in baseline toxicity. 


\section{Introduction}

Numerous scientific publications have suggested, reported, and discussed the existence of a generic cut-off in baseline toxicity based on the hydrophobicity of organic compounds (Könemann, 1981; Connell and Hawker, 1988; Barron, 1990; Meylan et al., 1999; Mayer and Reichenberg, 2006; Jonker and van der Heijden, 2007; Mayer and Holmstrup, 2008; Kwon et al., 2016). Using the octanol to water partition coefficient $\left(\mathrm{K}_{\mathrm{OW}}\right)$ to express hydrophobicity, a generic cut-off in baseline toxicity has been reported at $\log \mathrm{K}_{\mathrm{OW}} \approx 6$ by Könemann (1981) based on tests with 72 industrial compounds and guppies, at $\log \mathrm{K}_{\mathrm{OW}} \approx 6$ by Connell and Hawker (1988) based on tests with 42 chlorinated hydrocarbons and guppies/goldfish, and at Log $\mathrm{K}_{\mathrm{OW}} \geq 6$ by Gobas and Mackay (1987) based on tests with 18 hydrophobic organic chemicals and rainbow trout. In aquatic environments, the existence of a generic hydrophobicity cut-off in baseline toxicity has been explained by the low - often perceived as negligible - aqueous concentrations of highly hydrophobic organic compounds (HOCs) in such systems, resulting from strong sorption and/or volatilization of these compounds (Veith et al., 1983; Hawker and Connell, 1985; Mayer and Reichenberg, 2006; Smith et al., 2010a; Kwon et al., 2016). To the contrary, more recently the baseline toxicity of HOCs (with Log $\mathrm{K}_{\mathrm{OW}} \geq 3$ ) has been reported to generally initiate at a chemical activity of 0.01 to 0.1 , with no apparent cut-off in toxicity with increasing hydrophobicity (Reichenberg and Mayer, 2006; Mayer and Holmstrup, 2008; Schmidt and Mayer, 2015). Chemical activity (a) quantifies the energetic level, and not the mass concentration, of a HOC relative to the energetic level in its pure liquid (reference state, $a=1$; Reichenberg and Mayer, 2006; Mayer and Holmstrup, 2008). The maximum chemical activity of a hydrophobic organic liquid is by definition 1 , when using the pure liquid phase as reference state, and these liquids are thus by default expected to have the potential for exerting baseline toxicity at saturation (Reichenberg and Mayer, 2006). 
To challenge the existence of a generic Log $\mathrm{K}_{\mathrm{Ow}}$ cut-off in baseline toxicity, reliable toxicity data are needed for HOCs with $\log \mathrm{K}_{\mathrm{OW}}>6$. To this date, aquatic toxicity testing of these compounds is associated with a range of technical challenges (Smith et al., 2010b; Smith et al., 2012; Vergauwen et al., 2015; Stibany et al., 2017; OECD, 2019). The current study provides a full base-set of ecotoxicological data for a highly hydrophobic organic liquid (dodecylbenzene, DDB, $\log \mathrm{K}_{\mathrm{OW}}=8.65$ ) as required according to $\mathrm{REACH}$ for the evaluation of an organic compound of concern (European Union, 2006). This is particularly important for highly hydrophobic compounds, as their physicochemical properties result in strong sorption and thereby high stability in aquatic environments. According to the criteria of Annex XIII of REACH (European Union, 2006), the combination of intrinsic hydrophobicity and potential toxicity generally qualifies these compounds as PBT/vPvB suspected substances (persistent, bioaccumulative, toxic / very persistent, very bioaccumulative). Here, the highly hydrophobic model compound DDB is tested with organisms spanning three trophic levels, specifically primary producers (algae), primary consumers (crustacean), and secondary consumers (fish), using a newly developed passive dosing methodology for testing hydrophobic organic liquids exactly at saturation (Stibany et al., 2017). The toxicity results on algae, crustacean, and fish are then used in a wider discussion of the toxicity associated with highly hydrophobic compounds and the proposed generic hydrophobicity cut-off in baseline toxicity.

A major challenge when experimentally testing the aquatic toxicity of HOCs is to establish and maintain the exposure throughout the test, and thereby avoid underestimating the toxicity of the compound. In 2017, the authors of the current study developed, optimized, and applied a novel passive dosing methodology, addressing the technical challenges of testing hydrophobic organic liquids (Stibany et al., 2017). In passive dosing, a polymer (e.g., silicone) is loaded with the test compound, added to the test system, and then applied to 
control the exposure of the compound by continuous equilibrium partitioning. In this way, the exposure is maintained as losses resulting from, e.g., sorption and evaporation are efficiently buffered by re-partitioning from the loaded polymer. Also, the passive dosing methodology does not require the addition of co-solvents, which are often used to spike test compounds to the exposure medium in current standard aquatic toxicity tests. In the 2017 study, a novel loading procedure was developed utilizing the high silicone swelling potential for hydrophobic organic liquids (Stibany et al., 2017). Silicone O-rings were saturated by direct immersion in the pure liquid test substance, which resulted in a swelling exceeding $14 \%$ for DDB (i.e., "loading by swelling"). The O-rings loaded to saturation were then applied to establish and maintain exposure of DDB exactly at its solubility limit in 72-h growth inhibition tests with green algae Raphidocelis subcapitata. Growth rate inhibition of DDB at solubility was $13 \pm 5 \%(95 \% \mathrm{CI})$ in a first and $8 \pm 3 \%$ (95\% CI) in a repeated test, which demonstrated that improved exposure control can lead to good precision and repeatability of toxicity tests. Furthermore, a moderate toxicity at chemical activity of unity was higher than expected relative to reported hydrophobicity cut-offs in baseline toxicity, but lower than expected relative to the reported chemical activity range for baseline toxicity (Stibany et al., 2017).

Based on these 2017 findings (Stibany et al., 2017) and the reported chemical activity range for initiation of baseline toxicity $(\mathrm{a}=0.01-0.1)$, the main hypothesis of the current study is that hydrophobic organic liquids may exert baseline toxicity to aquatic organisms of all trophic levels when tested at the maximum exposure level (i.e., the solubility limit, chemical activity of unity). To obtain the full base-set of aquatic toxicity data for the highly hydrophobic liquid DDB, the novel passive dosing methodology (i.e., "loading by swelling") was adapted to and applied in an extended set of ecotoxicological test systems for toxicity testing exactly at the saturation level. Specifically, in the current study Daphnia magna acute 
immobilization (OECD, 2004) and Danio rerio fish embryo acute toxicity tests (FET)

124

125

126

127

(OECD, 2013) were conducted in addition to the already reported algae growth inhibition tests (Stibany et al., 2017). Unlike the algal growth inhibition test, both Daphnia

immobilization and FET tests are static systems with no agitation of the test system. In case of the Daphnia immobilization test, this required an optimization of the methodology towards a silicone O-ring enclosure system (cage) to separate the organisms from the dosing polymer, and thereby avoid direct contact exposure.

A Log $\mathrm{K}_{\mathrm{OW}}$ of 8.65 has been determined and reported for DDB based on measured HPLC retention times relative to different alkyl benzenes with experimental $\log \mathrm{K}_{\mathrm{OW}}$ values (Sherblom et al., 1992). This value is in reasonable agreement with predicted Log $\mathrm{K}_{\mathrm{OW}}$ values for DDB of 7.94 (EPISuite, U.S. EPA, USA) and 8.52 (ACD/Labs, Canada). Also, DDB has a melting point of $7^{\circ} \mathrm{C}$ and thus exemplary represents the properties of highly hydrophobic liquids. Furthermore, only few aquatic toxicity data on DDB can be found in the literature (Gledhill et al., 1991; Stibany et al., 2017). DDB itself is used as a cooling lubricant for machining processes in the metalworking industry (Baumann and Herberg-Liedtke, 1995). However, its most important application is in large scale production of linear alkylbenzene sulfonates, e.g., sodium dodecylbenzene sulfonate (Kosswig, 2000; Johansson and Somasundaran, 2007). Linear alkylbenzene sulfonates are regarded the most important group of anionic surfactants, with a global production volume in the range of $3000 \mathrm{kT}$ annually (Johansson and Somasundaran, 2007; HERA, 2013), and they are widely used in cleaning agents, laundry detergents, personal care products (Kosswig, 2000), and as emulsifiers in pesticide formulations (Mueninghoff et al., 2001; Knowles, 2012). The production of linear alkylbenzene sulfonates is a major anthropogenic source of DDB to the environment (Gledhill et al., 1991; Takada and Eganhouse, 1998; Dwiyitno et al., 2016). Reported environmental concentrations of DDB range from up to $0.2 \mu \mathrm{g} \mathrm{g}^{-1}$ sediment (dw) in German river systems 
(e.g., Lippe river)(Heim et al., 2004) to up to $99 \mu \mathrm{g} \mathrm{g}^{-1}$ sediment (dw) in the surroundings of megacities (e.g., Jakarta Bay)(Dwiyitno et al., 2016). Consequently, DDB has been suggested and used as a molecular marker for monitoring anthropogenic activities (Takada et al., 1990).

\section{Materials and Methods}

\subsection{Chemicals and materials}

Analytical grade dodecylbenzene (DDB, $99.8 \%$ according to certificate of analysis) was used as model compound and acquired from TCI Europe N.V., Belgium. Food-grade silicone Orings were used as partitioning donors in the initial passive dosing experiments, the Daphnia magna immobilization tests (Daphnia tests), and the Danio rerio fish embryo toxicity tests (FET tests). The O-rings used for the Daphnia tests had a cross section of $5.33 \mathrm{~mm}$, an outer diameter of $21.13 \mathrm{~mm}$, and a mass of $1452.5 \pm 20.1 \mathrm{mg}($ mean $\pm S D, n=20)(A l t e c$ Products Ltd, United Kingdom, product code ORS-BS309). The O-rings used for the FET tests had a cross section of $2.62 \mathrm{~mm}$, an outer diameter of $47.76 \mathrm{~mm}$, and a mass of $991.1 \pm 7.1 \mathrm{mg}(\mathrm{mean} \pm \mathrm{SD}$, n=10)(Altec Products Ltd, United Kingdom, product code ORS-BS131). Stainless steel was used for the enclosure system (cage) applied in a subset of the Daphnia tests (CNC milled and equipped with stainless steel grids by in-house workshop, RWTH Aachen University, Germany). Daphnia and FET tests were conducted in 100-mL glass bottles equipped with PTFE-lined screw caps. To minimize DDB sorption, only inert materials (e.g., glass, stainless steel, and PTFE) were used in contact with exposure media and organisms. All glassware was rinsed twice with methanol, ethanol, and water before use. A complete list of materials is provided in the SI (Table S1). 


\subsection{Pre-test procedures}

The procedures described in this paragraph were conducted prior to all tests in the current study, with further details provided in Stibany et al. (2017). Briefly, the O-rings were precleaned for two hours in excess methanol, overnight in excess methanol, and for two hours in excess ethanol. After cleaning, the O-rings were rinsed in purified water and left to dry for at least $7 \mathrm{~d}$ at $20.0 \pm 0.5^{\circ} \mathrm{C}$. Then, the O-rings were loaded by immersion in excess pure liquid DDB at room temperature for $24 \mathrm{~h}$ in the dark (i.e., loading by swelling). After loading, the O-rings were carefully rinsed twice with purified water and dried with lint-free tissues. Precleaned, unloaded O-rings (to be used in controls) were rinsed and dried in the same way (Smith et al., 2010b). Finally, one DDB loaded O-ring was added to each 100-mL test bottle containing $50 \mathrm{~mL}$ test medium. DDB was pre-equilibrated between the loaded silicone polymer and the test medium for $48 \mathrm{~h}$ at $300 \mathrm{rpm}$ (in the dark) before test start (Stibany et al., 2017). The temperatures during pre-equilibration and tests were $20 \pm 0.5^{\circ} \mathrm{C}$ (Daphnia) and $26 \pm 1^{\circ} \mathrm{C}(\mathrm{FET})$.

\subsection{Exposure confirmation}

Prior to toxicity testing, exposure at saturation was confirmed throughout a $96-\mathrm{h}$ period in separate tests. For these exposure confirmation tests, 15 loaded O-rings were individually preequilibrated in test medium to be used in Daphnia test and 15 loaded O-rings were individually pre-equilibrated in test medium to be used in FET tests, as described above (specifications of the media are provided in sections 2.4. and 2.5.). At each sampling time (0, 24, 48, 72, and $96 \mathrm{~h}$ after pre-equilibration), a 30-mL sample was collected from each replicate $(n=3)$ and the test bottle was discarded. For exposure confirmation in Daphnia cage tests (see below), 9 loaded O-rings were enclosed in the cages after pre-equilibration and sampling was performed 24, 48, and $96 \mathrm{~h}(\mathrm{n}=3)$ thereafter. All samples were extracted and 
analyzed by GC-MS (methods are provided in section 2.6.) to determine the freely dissolved concentrations of DDB.

\subsection{Acute Daphnia immobilization tests}

The acute Daphnia magna immobilization tests were conducted according to OECD guideline 202 (OECD, 2004) using Elendt M4 standard medium. Deviating from the guideline, Daphnia tests were prolonged to $72 / 96 \mathrm{~h}$.

To prevent direct contact between the migrating daphnids and the silicone O-rings, whilst allowing for undisturbed equilibrium passive dosing, the test system was optimized by the development of an O-ring enclosure system (cage). The cage was entirely made from stainless steel and consisted of a cylindrical base covered by a grid with a mesh size of $200 \mu \mathrm{m}$ (see Figure 2B).

For production of neonate daphnids, single adult D. magna were kept in $20 \mathrm{~mL}$ Elendt M4 medium (OECD, 2004) at $20^{\circ} \mathrm{C}$ and fed on the algae Desmodesmus subspicatus three times a week. A constant light-dark rhythm of $16: 8 \mathrm{~h}$ was maintained, and the medium was renewed on a weekly basis. At test start, five neonate daphnids $(<24 \mathrm{~h})$ were added to each replicate and pre-equilibrated test bottle (see section 2.2.). In the Daphnia cage tests, all O-rings (including the controls) were enclosed in the cages after pre-equilibration and before adding the daphnids. All test bottles were then kept fully randomized at experimental conditions under a light-dark regime of 16:8 h. Each Daphnia test consisted of 5 treatments, 5 controls (including pre-cleaned, unloaded O-rings and O-ring cages if applicable), and 5 global controls (only medium). In total, three Daphnia tests and six Daphnia cage tests were conducted. $\mathrm{O}_{2}$ level and $\mathrm{pH}$ were measured in three global controls and three treatments at test start and at the end of the test. All validity criteria were met for all nine tests, according to OECD guideline 202. Daphnia immobilization was assessed after 24, 48, 72, and $96 \mathrm{~h}$ according to the guideline (OECD, 2004). 
228

229

The acute FET tests were conducted according to OECD guideline 236 (OECD, 2013) using standard medium.

Zebrafish embryos were provided by the Fraunhofer Institute for Molecular Biology and Applied Ecology (IME), Aachen, Germany. Adult zebrafish were kept in breeding groups of $300-400$ individuals in a 250-L glass tank. The water was cleaned by circulation through an UV- and activated-carbon filter. The fish were fed twice daily with TetraMin dry flakes (Tetra $\mathrm{GmbH}$, Melle, Germany) and additionally with live Nauplius larvae of Artemia spp. (Great Salt Lake Artemia, Sanders, UT, USA). A constant light-dark rhythm of 14:10 h and a temperature of $26 \pm 2{ }^{\circ} \mathrm{C}$ was maintained. Breeding dishes were placed in the glass tanks just after the light was switched on in the morning and collected $1 \mathrm{~h}$ later. Immediately after collection, the eggs were rinsed with water, transferred to a crystallization dish filled with standard medium according to OECD guideline 236 (OECD, 2013), and inspected for viability. At test start, five fish eggs (max. 3 hours post fertilization; hpf) were added to each replicate and pre-equilibrated test bottle (see section 2.2.). All test bottles were then kept fully randomized at experimental conditions. FET tests were conducted in the dark (except during the assessment of effects). Each test consisted of 5 treatments, 5 controls, and 5 global controls. In total, two FET tests were conducted. $\mathrm{O}_{2}$ level and $\mathrm{pH}$ were measured in three global controls and three treatments at test start and at the end of the test. All validity criteria were met for both tests, according to OECD guideline 236.

Fish embryo lethality was assessed under the microscope (Nikon Eclipse TS100) after 24, 48, 72, and $96 \mathrm{~h}$ according to the guideline (OECD, 2013). Each egg was considered individually. Lethal effects included: egg coagulation, no tail detachment, no somite formation, and no heartbeat. Sublethal effects after $24 \mathrm{~h}$ included: incomplete epiboly, growth retardation, and no spontaneous movement, while sublethal effects after $48 \mathrm{~h}$ included: no eye development, 
weak heartbeat, weak blood circulation, no/weak pigmentation, oedema, malformations, modified structure of the chorda, and growth retardation.

\subsection{Extraction and GC-MS analysis}

Extraction of the 30-mL medium samples from the exposure confirmation tests (section 2.3.) was performed by addition of $15 \mathrm{~mL}$-hexane (HPLC-grade, VWR International GmbH, Darmstadt, Germany), followed by 5 min shaking by hand, and 20 min shaking at $280 \mathrm{rpm}$ on a shaker. After separation and collection of the organic extract, the extraction procedure was repeated. The two extracts were combined, and $0.6 \mathrm{~mL}$ toluene (HPLC-grade, VWR International GmbH, Darmstadt, Germany) was added. The n-hexane was then completely evaporated by rotary evaporation at $40^{\circ} \mathrm{C}$ and 120 mbar (Laborota 4011 Digital, Heidolph Instruments, Schwabach, Germany). The toluene extract was restocked to $1 \mathrm{~mL}$ and placed in GC vials until chemical analysis. Acceptable recovery during the extraction process was confirmed by adding a defined amount of DDB (i.e., $1 \mu \mathrm{g}$ ) to n-hexane before rotary evaporation of the solvent, restocking with toluene, and measuring by GC exactly as described above (percentage recovery: $95 \pm 4 \%$, mean $\pm \mathrm{SD}, \mathrm{n}=5$; data not shown).

GC-MS (Agilent GC 6890N) was used to quantify DDB. Measurements were conducted in SIM mode at 92 and $246 \mathrm{~m} / \mathrm{z}$. A sample volume of $2 \mu \mathrm{L}$ was injected in splitless mode on an Optima® 35 MS 0.25 mm, 30 m column (Agilent Technologies, Palo Alto, CA, USA). The carrier gas was helium and was operated at a flow rate of $0.8 \mathrm{~mL} \mathrm{~min}^{-1}$. The following temperature program was used: $60^{\circ} \mathrm{C}$ between 0 and 2 min, then constant heating of $10^{\circ} \mathrm{C}$ $\min ^{-1}$ up to $340^{\circ} \mathrm{C}$, which was finally kept constant for $5 \mathrm{~min}$. DDB concentrations were quantified by a 13-point external standard calibration curve fitted with a second order polynomial regression. Signal integration was performed by Chemstation software (B.03.01, Agilent Technologies, Palo Alto, CA, USA). 
To identify possible outliers, the Hampel outlier test was performed on the exposure all sampling times were checked for normal distribution (Kolmogorov-Smirnov Test) and variance homogeneity (Levene's Test) and then analyzed by Student's t-test or Welch's t-test, depending on the statistical characteristics (ToxRat 2.10, ToxRat Solutions GmbH, Alsdorf, cage tests, each test system was first checked separately for significant differences between the replicates (one-way ANOVA with a post-hoc Tukey's range test). Second, in case of no significant difference within each of the two test systems, data from each system were pooled and the two test systems compared by Student's t-test (GraphPad Prism 6.01, GraphPad tests. All graphs were designed with GraphPad Prism software.

\section{Results and Discussion}

\subsection{Passive dosing and exposure confirmation}

In recent years, several studies and publications have addressed the use of passive dosing for ecotoxicological investigations of both solid and liquid HOCs (Mayer and Holmstrup, 2008; Smith et al., 2010a; Kwon and Kwon, 2012; Stibany et al., 2017; Trac et al., 2018). In our study from 2017 (Stibany et al., 2017), we demonstrated that silicone O-rings are well suited as a passive dosing donor, especially for liquid HOCs to be tested exactly at their solubility limit (saturation limit). We utilized the high capacity of the silicone to take up HOCs, and 
direct immersion in the pure liquid HOC was applied to load the silicone to the saturation level (i.e., "loading by swelling"). The method was validated and applied in the algae growth inhibition test based on OECD guideline 201 (OECD, 2011; Stibany et al., 2017).

Furthermore, the passive dosing methodology was included in the updated OECD guideline 23 (OECD, 2019). Some key data on the methodology were collected in the 2017 study (Stibany et al., 2017). First, for DDB the total uptake capacity of several types of silicone Orings along with the uptake kinetics were determined (capacity for DDB $=14.5 \pm 0.2 \mathrm{wt} \%$ $[$ mean $\pm \mathrm{SD}, \mathrm{n}=48]$; max. time to saturation $\mathrm{t}_{95 \%}=16.5 \mathrm{~h}$ ). Second, the time to reach the equilibrium concentration in algal test medium was determined $\left(\mathrm{t}_{95 \%}=10.8 \mathrm{~h}\right)($ Stibany et al., 2017). These results were in line with reported results for the equilibration of polycyclic aromatic hydrocarbons between loaded polymers and water as well as cell culture medium (Smith et al., 2010b).

In the present study, the same materials and silicone loading procedure were applied for comparability, and our main technical aim was to extend the applicability of this methodology by adapting, validating, and applying the method to other test systems and test species. The first step was then to confirm the stability of DDB exposure at saturation in the Daphnia and Fish Embryo Acute Toxicity (FET) tests. DDB exposure concentrations and stability were determined for the test duration of 96 hours. In the Daphnia medium, a mean DDB concentration of $15.7 \pm 2.9 \mu \mathrm{g} \mathrm{L}^{-1}$ (mean $\pm \mathrm{SD}, \mathrm{n}=3$ ) was measured between 0 and $96 \mathrm{~h}$ (Figure 1A), whereas it was $16.9 \pm 1.9 \mu \mathrm{g} \mathrm{L}^{-1}$ (mean $\pm \mathrm{SD}, \mathrm{n}=3$ ) between 24 and $96 \mathrm{~h}$ in Daphnia medium in the presence of O-ring cages (Figure 1B). The mean concentration in the FET medium was $12.2 \pm 2.8 \mu \mathrm{g} \mathrm{L}^{-1}$ (mean $\pm \mathrm{SD}, \mathrm{n}=3$ ) between 0 and $96 \mathrm{~h}$ (Figure $1 \mathrm{C}$ ). In all cases, exposure concentrations were constant throughout the test durations. Also, all measured exposure concentrations were in the same range as reported for algal medium in our previous study (i.e., a mean DDB concentration of $12 \pm 5 \mu \mathrm{g} \mathrm{L}{ }^{-1}$, mean $\pm \mathrm{SD}, \mathrm{n}=4$ )(Stibany et al., 2017). 

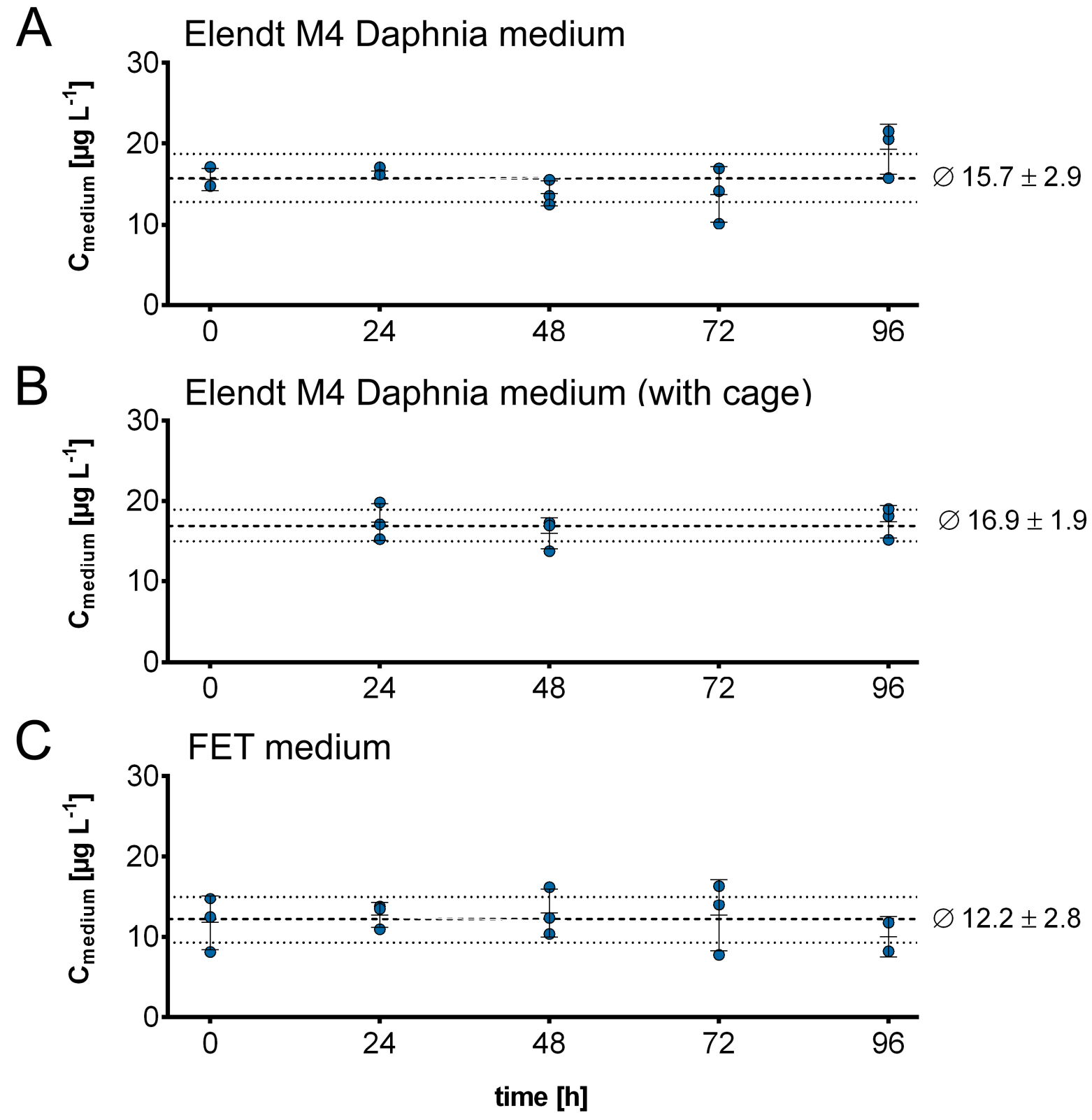

Figure 1: Dodecylbenzene (DDB) exposure concentrations after 48-h pre-equilibration plotted against time in the respective test medium, i.e., A) Elendt M4 Daphnia medium, B) Elendt M4 Daphnia medium with the silicone O-rings enclosed in cages, and C) FET medium. Each data point represents a single measurement. Additionally, mean \pm SD ( $n=3$ ) are given for each sampling. The dotted lines show the overall mean concentration from $0 \mathrm{~h}$ to $96 \mathrm{~h}$ (mean $\pm \mathrm{SD}$ ). All data in the graphs are provided in the SI (Table S2).

Overall, the applied passive dosing format was found to be a suitable technique in this first validation step. The approach is effective in terms of handling and performing well for the 
challenging and highly hydrophobic model compound DDB. Constant exposure concentrations at the solubility limit were achieved in all test systems with algae (Stibany et al., 2017), daphnids and fish embryos. Furthermore, the data are in good agreement with reputable prediction tools. A DDB water solubility of $7.8 \mu \mathrm{g} \mathrm{L}^{-1}\left(31.7 \mathrm{nmol} \mathrm{L}{ }^{-1}\right)$ was predicted with ACD/I-Labs, while an EPI Suite ${ }^{\mathrm{TM}}$ query resulted in a water solubility range of 1.0 to 1.7 $\mu \mathrm{g} \mathrm{L}^{-1}$ (4.1 to $6.9 \mathrm{nmol} \mathrm{L}^{-1}$ ) depending on the algorithm used (ACD/Labs, 2016; US EPA, 2016). Taking into consideration experimental and analytical challenges related to solubility and exposure determinations in the lower $\mu \mathrm{g} \mathrm{L}^{-1}$ range (Birch et al., 2019), the differences between measurements and predictions appear acceptable. Overall, the "loading by swelling" passive dosing methodology and pre-equilibration procedures were consistent and fully supported the application, not only in algae tests but also in multiple ecotoxicity test systems with other test organisms.

\subsection{Aquatic toxicity}

After the technical adaptation and validation of the passive dosing format, which was found to be fully compatible with the Daphnia immobilization and the FET test, the second step was to apply the system in actual toxicity assays. The Daphnia magna immobilization test was first performed in a manner comparable to the algal growth inhibition test (Stibany et al., 2017), except the test system was operated in a static mode. At a freely dissolved DDB concentration of $15.7 \pm 2.9 \mu \mathrm{g} \mathrm{L}^{-1}$ (mean $\pm \mathrm{SD}, \mathrm{n}=3$; according to the exposure confirmation, Figure $1 \mathrm{~A}$ ), the daphnid immobilization after $72 \mathrm{~h}$ was $72 \pm 5 \%$ in a first test, $64 \pm 10 \%$ in a second test, and $80 \pm 6 \%$ in a third test (mean \pm SEM)(Figure $2 \mathrm{~A}$, light blue bars). However, it was observed that almost all immobilized daphnids adhered to the silicone O-rings, some individuals displaying weak vital signs. This was not the case in the controls with unloaded O-rings nor in the global controls without O-rings. It was not possible to differentiate whether the immobilized daphnids adhered to the polymer due to an adhesive effect of DDB present on the surface of 
the O-ring or if they were immobilized by the freely dissolved test substance. According to OECD guideline 202 (OECD, 2004), daphnid individuals that are unable to swim within 15 seconds, after gentle agitation of the test vessel, are considered immobilized. It is not necessary to determine/specify the exact cause of immobilization for an experiment to be considered valid. In the present study, however, we aimed to achieve a sensitive test system with a clear link between the freely dissolved DDB concentration and the resulting effect for distinct ecotoxicological data. Several strategies to remove excess DDB, potentially present on the O-ring surface, were tested to decrease the observed adherence of the daphnids to the polymer. These strategies included methanol rinsing and cleaning in water during sonication (data not shown). None of these procedures led to a decreasing adhesion effect. At the same time, any type of cleaning strategy for the fully loaded O-ring represents a high risk of effecting the saturation of the polymer and thus the resulting exposure concentration. To avoid these possible side effects, stainless steel cages were developed to house a single loaded O-ring (Figure 2B), which could then be inserted into a test vessel without additional cleaning steps. In this way, the adhesion of the daphnids to the polymer, i.e., the direct contact of the animals with the polymer surface, was prevented without influencing the saturation of the Orings and the release of the test substance to the exposure medium (Figure 2B).

The cages were applied in a total of six Daphnia magna immobilization tests, called Daphnia cage tests. At a freely dissolved DDB concentration of $16.9 \pm 1.9 \mu \mathrm{g} \mathrm{L}^{-1}($ mean $\pm \mathrm{SD}, \mathrm{n}=3$; according to the exposure confirmation, Figure 1B), the treatments showed increasing immobilization over time with up to $32 \pm 10 \%$ in the first test, $32 \pm 8 \%$ in the second test, $12 \pm 8 \%$ in the third test, $8 \pm 5 \%$ in the fourth test, $20 \pm 9 \%$ in the fifth test, and $12 \pm 8 \%$ in the sixth test (mean \pm SEM) after $72 \mathrm{~h}$ (Figure 2A, dark blue bars). In general, the immobilization was significantly reduced compared to the tests without cages after both 48 and $72 \mathrm{~h}$ (p < 0.0001). Due to the application of the cages, adhesive effects of the O-rings or DDB 
potentially present on the O-ring surface could be excluded. In these tests, the immobilization of daphnids was thus directly related to the freely dissolved concentration of DDB in the test medium. The Daphnia cage tests were conducted over a time span of $96 \mathrm{~h}$. However, the immobilization in several controls and global controls exceeded the validity criteria at $96 \mathrm{~h}$ (controls are not valid if immobilization $>10 \%$; Figure 2A). Thus, data on daphnia immobilization by DDB were only regarded valid within the first $72 \mathrm{~h}$ of all tests.

The FET tests were performed in a manner comparable to the algal growth inhibition tests from 2017 (Stibany et al., 2017), except the test system was operated in a static mode. Since the risk of adhesion of the test organisms to the polymer surface was found very low in this test system (static test system and immobile organisms), an O-ring cage was not applied. At a freely dissolved DDB concentration of $12.2 \pm 2.8 \mu \mathrm{g} \mathrm{L}^{-1}$ (mean $\pm \mathrm{SD}, \mathrm{n}=3$; according to the exposure confirmation, Figure 1C), no lethal effects were observed within $96 \mathrm{~h}$ in any of the two FET tests conducted (Table S3). Sub-lethal effects, i.e., malformations, no/weak pigmentation, and oedema were sporadically observed in single embryos in one FET test after $96 \mathrm{~h}$ (data provided in Table S3). These sublethal effects were found to be negligible. No (sub)lethal effects were observed within the test duration of $96 \mathrm{~h}$ in the controls nor the global controls of the FET tests.

Overall, the precision and the repeatability of test results were high for all applied test systems adapted to the new passive dosing format. No statistically significant differences were observed between the three Daphnia tests $(\mathrm{p}>0.88 ; 72 \mathrm{~h})$ respectively the six Daphnia cage tests $(\mathrm{p}>0.46 ; 72 \mathrm{~h})$. As presented in the 2017 paper, the results of the algal growth inhibition tests showed very high precision and repeatability (Stibany et al., 2017). 


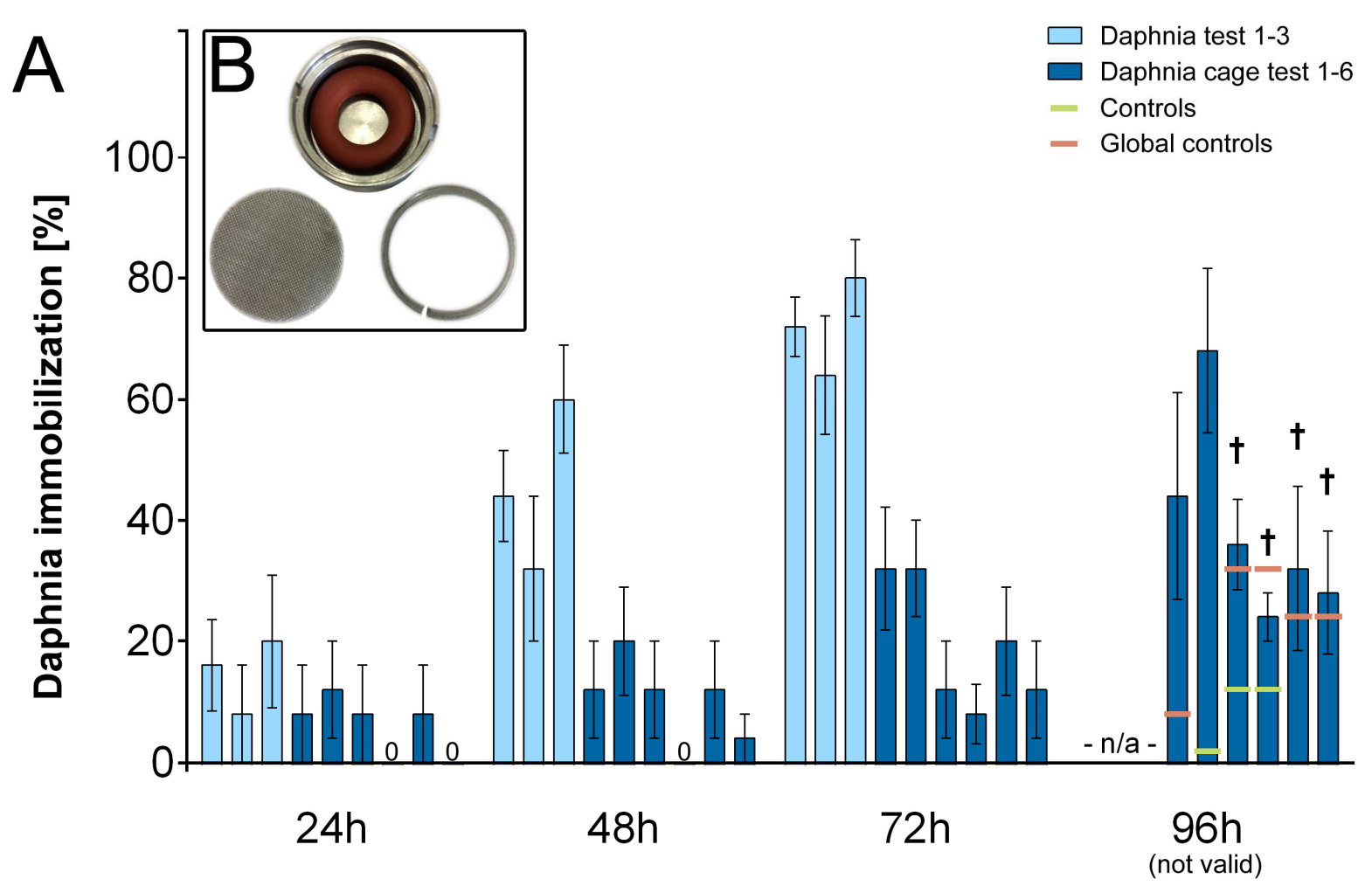

sampling time

Figure 2: Daphnia immobilization tests. A) Three conducted Daphnia tests (light blue bars) and six Daphnia cage tests

(dark blue bars) with dodecylbenzene (DDB) exposure exactly at the solubility limit. Each bar represents the

immobilization mean $\pm \operatorname{SEM}(\%, \mathrm{n}=5)$ in a single Daphnia magna immobilization test at the respective sampling time $(24$,

48,72 , and $96 \mathrm{~h}$ ). The mean control immobilization (>0) is shown as green dashes, the mean global control

immobilization (>0) is shown as orange dashes. The 96-h sampling was conducted in the Daphnia cage tests only.

Controls and global controls exceeding the test validity criteria are marked with crosses. B) Photo of a silicone O-ring

cage containing an O-ring, top grid, and closure ring. All data in the graphs are provided in the SI (Table S4).

During the assessment of DDB ecotoxicity towards aquatic organisms, we observed clear differences in the base-set of ecotoxicological data presented in the current study. As reported, 427 the growth rate inhibition of DDB to algae at the solubility limit was $13 \pm 5 \%(95 \% \mathrm{CI})$ in a

428 first and $8 \pm 3 \%(95 \% \mathrm{CI})$ in a repeated test (Stibany et al., 2017). This moderate toxicity was higher than expected relative to reported hydrophobicity cut-offs in baseline toxicity 
Reichenberg, 2006; Jonker and van der Heijden, 2007; Mayer and Holmstrup, 2008; Kwon et

al., 2016). The Daphnia tests showed substantial immobilization effects, also after linking these effects exclusively to the freely dissolved concentrations of DDB (by introducing the Oring cage). Even though no effects were observed in the FET tests, the fact that two out of three species were affected by DDB clearly indicates a certain toxicity of this compound with a $\log \mathrm{K}_{\mathrm{OW}}>$ 8. Comparing these results to results found in the literature is not straightforward, as reliable literature data are scarce. Few reported toxicity data are available on different mixtures of linear alkyl benzenes (LABs) at undefined exposure concentrations (mean alkyl chain lengths of C11-C13, thus including DDB) published by Gledhill et al. (1991). Depending on the applied LAB mixture, an $\mathrm{EC}_{50}$ of 9-80 $\mu \mathrm{g} \mathrm{L}^{-1}$ was reported for daphnids after $48 \mathrm{~h}$. However, most of the reported concentration range exceeded the solubility of $41 \mu \mathrm{g} \mathrm{L}^{-1}$ reported by Gledhill et al. (1991) as well as the solubility limits measured in the present study (see section 3.1.). Furthermore, Gledhill et al. (1991) did not observe acute or chronic effects in other invertebrates, fish, or algae. Regarding the absence of toxic effects in the FET tests in the present study, the literature does provide some indication. It has been suggested, that the chorion of the fish embryo could act as a barrier for uptake of chemicals and that fish provide proper metabolism and/or elimination capabilities related to LABs, including DDB (Gledhill et al., 1991; Henn and Braunbeck, 2011; Kais et al., 2013). However, it is also possible that the test duration was too short to account for the potentially slow uptake kinetics of DDB in fish (through the chorion), especially as the system was static (unstirred) (Butler et al., 2016; Mayer and Schmidt, 2017). Hence, a toxicity of DDB to fish can neither be confirmed nor excluded based on the results reported here.

\subsection{Major implications}

The aims of this study were to further improve our new passive dosing format for robust exposure control in aquatic toxicity tests and to provide reliable aquatic toxicity data for a 
highly hydrophobic model compound. Concluding with the current study, we provide a full base-set of reliable ecotoxicological data on DDB, as needed for the risk evaluation of a chemical under the European REACH regulation (European Union, 2006).

\section{The main hypothesis of the current study was that hydrophobic organic liquids may exert} baseline toxicity to aquatic organisms of all trophic levels when tested at the maximum exposure level (i.e., the solubility limit, chemical activity of unity). Our results for DDB showed moderate toxicity towards algae (Stibany et al., 2017), moderate toxicity towards daphnids, and no observed toxicity to fish embryos. On one hand, toxicity of DDB was not expected based on the suggested Log $\mathrm{K}_{\mathrm{OW}}$ cut-off in baseline toxicity for substances with a Log $\mathrm{K}_{\mathrm{OW}}>$ 5-6 (Könemann, 1981; Barron, 1990). Taking into account the exceedingly high hydrophobicity of DDB (Log $\left.K_{\mathrm{OW}}=8.65\right)$ (Sherblom et al., 1992), the observed toxicity in two of three tested species clearly questions the existence of a generic Log $\mathrm{K}_{\mathrm{OW}}$ cut-off in baseline toxicity. Together with other studies (Reichenberg and Mayer, 2006; Mayer and Holmstrup, 2008; Schmidt and Mayer, 2015; Stibany et al., 2017; Gobas et al., 2018; Trac et al., 2018), these results form a serious burden of proof against this generalized theory, which for a long time has been a well-established doctrine. On the other hand, by definition DDB as a liquid has a maximum chemical activity of 1 . Based on the reported chemical activity range for the initiation of baseline toxicity $(\mathrm{a}=0.01-0.1)$, toxicity was expected to occur to a markedly higher extent (Mayer and Reichenberg, 2006; Mackay et al., 2009; Schmidt and Mayer, 2015; Gobas et al., 2018; Schmidt et al., 2018). In our studies, only moderate toxicity was observed towards two of three tested species.

Considering these implications and results from other published studies (Mackay et al., 1980; Birch et al., 2019), Kow seems problematic as a measure/parameter for hydrophobicity when establishing a link between hydrophobicity and cut-offs in baseline toxicity for the following reasons: (1) the $\mathrm{K}_{\mathrm{OW}}$ lacks a mechanistic basis, since chemicals with high molecular weights 
tend to have a lower solubility in both octanol and water (Mackay et al., 1980; Birch et al., 2019), (2) expressing toxicity cut-offs on a $\mathrm{K}_{\mathrm{OW}}$ basis is counterintuitive, as baseline toxicity generally increases with increasing $\mathrm{K}_{\mathrm{OW}}$ due to increasing water to lipid partitioning and then suddenly the compounds become non-toxic (cut-off in toxicity), and (3) the $\mathrm{K}_{\mathrm{OW}}$ does not address the melting point effect on water solubility, i.e., the melting enthalpy required to transfer the solid chemical to a liquid state (Yalkowsky et al., 1979; Birch et al., 2019). One solution would be to address hydrophobicity based solely on water solubility. Water solubility sets the maximum concentration in the medium and the maximum gradient of the aqueous boundary diffusion gradient. With regards to water solubility it is more reasonable to expect a cut-off since a certain minimum concentration gradient might be needed to drive a sufficient diffusive flux into the organism to reach a critical body burden (CBB) needed to exert toxicity (Birch et al., 2019). CBBs and critical membrane concentrations for baseline toxicity are normally expressed on a molar basis, which in turn suggests a water solubility cut-off on a molar basis.

Methodologically, we successfully developed, adapted, validated, and applied the "loading by swelling" passive dosing technique to organisms of three different trophic levels, i.e., algae (primary producers), crustaceans (primary consumers), and fish (secondary consumers). Altogether, the method allowed for the determination of acute and chronic effects as well as lethal and sublethal endpoints. The observations from this study support the versatility, precision, and reproducibility of the novel passive dosing technique "loading by swelling" for testing liquid HOCs. The adaptation of both the Daphnia and the FET test was found to be straightforward, resulting in well-defined constant exposure concentrations and sensitive toxicity measurements (Figures 1 and 2). The new format proved to be an effective approach for the toxicity testing of this important group of chemicals and can provide the basis for more reliable risk assessments. Furthermore, it can act as a suitable extension to our 2017 method included in the new OECD guideline 23 (Stibany et al., 2017; OECD, 2019). The combination 
of improved exposure control via passive dosing, prolongation of the test durations, and the use of closed test systems provided good precision, while avoiding the use of co-solvents. Especially for HOCs, it has been widely discussed whether insufficient exposure time of short acute tests might lead to the underestimation of HOC toxicity (Mayer and Reichenberg, 2006; Smith et al., 2010a; Kwon et al., 2016). Of course, prolongation is limited in static test systems due to limited availability of nutrients and oxygen. In this study, this was illustrated by the invalidity of the Daphnia tests when prolonged to more than $72 \mathrm{~h}$. Still, the application of closed test systems is highly recommended, as many HOCs exhibit high air water partition coefficients $\left(\mathrm{K}_{\mathrm{AW}}\right)$, affecting the concentration in aqueous media.

\section{Conclusion}

The results of this study have two major conclusions: 1) Including the experimental results of this study, together with its predecessor from 2017 (Stibany et al., 2017), and the work of other groups, there is increasing evidence against the existence of a generic Log $\mathrm{K}_{\mathrm{OW}}$ cut-off in baseline toxicity for hydrophobic chemicals with a Log $\mathrm{K}_{\mathrm{OW}}$ exceeding 5-6. 2) DDB exerts aquatic toxicity at the solubility limit. This limit concentration is above expected environmental concentrations, and the environmental risk might thus be lower. However, taking into account the high production volumes, the reported presence in environmental samples (Heim et al., 2004; Dwiyitno et al., 2016), the potential presence of „solubilizers““ like DOC or microbial rhamnolipids in the environment, and the inappropriate (standard) test systems of the current hazard and risk assessments for HOCs, the risk of underestimating the toxicity of other hydrophobic compounds (that might even be more toxic than DDB) should be recognized. The methods presented in this and other recent studies (e.g., Trac et al., 2019) extend the methodological toolbox and facilitate more thorough and reliable assessments and evaluations of highly hydrophobic compounds as requested in the new OECD guideline 23 (OECD, 2019). 
536 Supporting Information

537 The Supporting Information is available free of charge.

538

539 Acknowledgements

540 This research was performed in the frame of ECOSM (Damme et al., 2011), a joint project

541 funded by the German Federal Environmental Foundation (DBU) and the German Cosmetic,

542 Toiletry, Perfumery and Detergent Association (IKW). We thank the IKW consortium as well

543 as the German Federal Environment Agency and ECT Oekotoxikologie GmbH for supporting

544 and accompanying the project. Philipp Mayer acknowledges additional funding from CEFIC-

545 LRI for the ECO38 project. Furthermore, we thank the group of Prof. Dr. Henner Hollert,

546 formerly at the RWTH Aachen University, for their support in conducting the fish-embryo

547 toxicity assay. 
ACD/Labs, 2016. ACD/I-Lab Online Property Prediciton, ilab.acdlabs.com. Advanced Chemistry Development, Inc., Toronto, Canada. Barron, M.G., 1990. Bioconcentration. Environ Sci Technol 24, 1612-1618.

Baumann, W., Herberg-Liedtke, B., 1995. Chemikalien in der Metallbearbeitung: Daten und Fakten zum Umweltschutz; mit 57 Tabellen. Springer.

Birch, H., Redman, A.D., Letinski, D.J., Lyon, D.Y., Mayer, P., 2019. Determining the water solubility of difficult-to-test substances: A tutorial review. Anal Chim Acta 1086, 16-28.

Butler, J.D., Parkerton, T.F., Redman, A.D., Letinski, D.J., Cooper, K.R., 2016. Assessing AromaticHydrocarbon Toxicity to Fish Early Life Stages Using Passive-Dosing Methods and Target-Lipid and Chemical-Activity Models. Environ Sci Technol 50, 8305-8315.

Connell, D.W., Hawker, D.W., 1988. Use of Polynomial Expressions to Describe the Bioconcentration of Hydrophobic Chemicals by Fish. Ecotox Environ Safe 16, 242-257.

Damme, S., Ratte, H.-T., Hollert, H., Coors, A., Knacker, T., Rettinger, K., Schulte, C., Schaffer, A., 2011. ECOSM-a new joint project for assessing environmental risks of poorly soluble compounds used in cosmetics-project presentation. Environmental Sciences Europe 23, 30.

Dwiyitno, Dsikowitzky, L., Nordhaus, I., Andarwulan, N., Irianto, H.E., Lioe, H.N., Ariyani, F., Kleinertz, S., Schwarzbauer, J., 2016. Accumulation patterns of lipophilic organic contaminants in surface sediments and in economic important mussel and fish species from Jakarta Bay, Indonesia. Mar Pollut Bull 110, 767-777.

European Union, 2006. Regulation (EC) No. 1907/2006 of the European Parliament and of the Council of 18 December 2006 concerning the Registration, Evaluation, Authorisation and Restriction of Chemicals (REACH).

Gledhill, W.E., Saeger, V.W., Trehy, M.L., 1991. An aquatic environmental safety assessment of linear alkylbenzene. Environ Toxicol Chem 10, 169-178.

Gobas, F.A.P.C., Mackay, D., 1987. Dynamics of Hydrophobic Organic-Chemical Bioconcentration in Fish. Environ Toxicol Chem 6, 495-504.

Gobas, F.A.P.C., Mayer, P., Parkerton, T.F., Burgess, R.M., van de Meent, D., Gouin, T., 2018. A Chemical Activity Approach to Exposure and Risk Assessment of Chemicals. Environ Toxicol Chem 37, 1235-1251.

Hawker, D.W., Connell, D.W., 1985. Relationships between Partition-Coefficient, Uptake RateConstant, Clearance Rate-Constant and Time to Equilibrium for Bioaccumulation. Chemosphere 14, 1205-1219.

Heim, S., Schwarzbauer, J., Kronimus, A., Littke, R., Woda, C., Mangini, A., 2004. Geochronology of anthropogenic pollutants in riparian wetland sediments of the Lippe River (Germany). Org Geochem 35, 1409-1425.

Henn, K., Braunbeck, T., 2011. Dechorionation as a tool to improve the fish embryo toxicity test (FET) with the zebrafish (Danio rerio). Comp Biochem Phys C 153, 91-98.

HERA, H.a.E.R.A.o.i.o.H.C.P., 2013. LAS Linear Alkylbenzene Sulphonate. HERA Report. Johansson, I., Somasundaran, P., 2007. Handbook for cleaning/decontamination of surfaces. Elsevier Science.

Jonker, M.T.O., van der Heijden, S.A., 2007. Bioconcentration factor hydrophobicity cutoff: An artificial phenomenon reconstructed. Environ Sci Technol 41, 7363-7369.

Kais, B., Schneider, K.E., Keiter, S., Henn, K., Ackermann, C., Braunbeck, T., 2013. DMSO modifies the permeability of the zebrafish (Danio rerio) chorion-Implications for the fish embryo test (FET). Aquat Toxicol 140-141, 229-238.

Knowles, A., 2012. Chemistry and Technology of Agrochemical Formulations. Springer Netherlands. Könemann, H., 1981. Quantitative structure-activity relationships in fish toxicity studies. Part 1: Relationship for 50 industrial pollutants. Toxicology 19, 209 - 221.

Kosswig, K., 2000. Surfactants. Ullmann's Encyclopedia of Industrial Chemistry. Wiley-VCH Verlag $\mathrm{GmbH} \&$ Co. KGaA. 
Kwon, H.C., Kwon, J.H., 2012. Measuring aqueous solubility in the presence of small cosolvent volume fractions by passive dosing. Environ Sci Technol 46, 12550-12556.

Kwon, J.-H., Lee, S.-Y., Kang, H.-J., Mayer, P., Escher, B.I., 2016. Including Bioconcentration Kinetics for the Prioritization and Interpretation of Regulatory Aquatic Toxicity Tests of Highly Hydrophobic Chemicals. Environ Sci Technol 50, 12004-12011.

Mackay, D., Arnot, J.A., Petkova, E.P., Wallace, K.B., Call, D.J., Brooke, L.T., Veith, G.D., 2009. The physicochemical basis of QSARs for baseline toxicity. Sar Qsar Environ Res 20, 393-414.

Mackay, D., Bobra, A., Shiu, W.Y., Yalkowsky, S.H., 1980. Relationships between Aqueous Solubility and Octanol-Water Partition-Coefficients. Chemosphere 9, 701-711.

Mayer, P., Holmstrup, M., 2008. Passive dosing of soil invertebrates with polycyclic aromatic hydrocarbons: Limited chemical activity explains toxicity cutoff. Environ Sci Technol 42, 7516-7521.

Mayer, P., Reichenberg, F., 2006. Can highly hydrophobic organic substances cause aquatic baseline toxicity and can they contribute to mixture toxicity? Environ Toxicol Chem 25, 2639-2644.

Mayer, P., Schmidt, S.N., 2017. Comment on "Assessing Aromatic-Hydrocarbon Toxicity to Fish Early Life Stages Using Passive-Dosing Methods and Target-Lipid and Chemical-Activity Models". Environ Sci Technol 51, 3584-3585.

Meylan, W.M., Howard, P.H., Boethling, R.S., Aronson, D., Printup, H., Gouchie, S., 1999. Improved method for estimating bioconcentration/bioaccumulation factor from octanol/water partition coefficient. Environ Toxicol Chem 18, 664-672.

Mueninghoff, J.C., Viets, A.K., Downer, R.A., Pesticides, A.C.E.-O., Formulations, A.C.E.-o.P.S.E.-o.P., Systems, A., 2001. Pesticide Formulations and Application Systems: A New Century for Agricultural Formulations : Twenty First Volume. ASTM.

OECD, 2004. Test No. 202: Daphnia sp., Acute Immobilisation Test and Reproduction Test. OECD Guidelines for the testing of chemicals 202, 16 pages.

OECD, 2011. Test No. 201: Freshwater Alga and Cyanobacteria, Growth Inhibition Test. OECD Guidelines for the testing of chemicals 201, 25 pages.

OECD, 2013. Test No. 236: Fish Embryo Acute Toxicity (FET Test). OECD Guidelines for the testing of chemicals 236.

OECD, 2019. Guidance Document on Aquatic Toxicity Testing of Difficult Substances and Mixtures.

Reichenberg, F., Mayer, P., 2006. Two complementary sides of bioavailability: Accessibility and chemical activity of organic contaminants in sediments and soils. Environ Toxicol Chem 25, 12391245.

Schmidt, S.N., Armitage, J.M., Arnot, J.A., Mackay, D., Mayer, P., 2018. Linking algal growth inhibition to chemical activity: Excess toxicity below $0.1 \%$ of saturation. Chemosphere $208,880-886$.

Schmidt, S.N., Mayer, P., 2015. Linking algal growth inhibition to chemical activity: Baseline toxicity required $1 \%$ of saturation. Chemosphere $120,305-308$.

Sherblom, P.M., Gschwend, P.M., Eganhouse, R.P., 1992. Aqueous solubilities, vapor-pressures, and 1-octanol-water partition-coefficients for C9-C-14 linear alkylbenzenes. J Chem Eng Data 37, 394-399. Smith, K.E.C., Dom, N., Blust, R., Mayer, P., 2010a. Controlling and maintaining exposure of hydrophobic organic compounds in aquatic toxicity tests by passive dosing. Aquat Toxicol 98, 15-24. Smith, K.E.C., Oostingh, G.J., Mayer, P., 2010b. Passive dosing for producing defined and constant exposure of hydrophobic organic compounds during in vitro toxicity tests. Chem Res Toxicol 23, 5565.

Smith, K.E.C., Rein, A., Trapp, S., Mayer, P., Karlson, U.G., 2012. Dynamic passive dosing for studying the biotransformation of hydrophobic organic chemicals: Microbial degradation as an example. Environ Sci Technol 46, 4852-4860.

Stibany, F., Schmidt, S.N., Schäffer, A., Mayer, P., 2017. Aquatic toxicity testing of liquid hydrophobic chemicals - Passive dosing exactly at the saturation limit. Chemosphere 167, 551-558.

Takada, H., Eganhouse, R.P., 1998. Molecularmarkers of anthropogenicwaste. in: Meyers, R.A. (Ed.). Encyclopedia of Environmental Analyses and Remediation John Wiley \& Sons, pp. 2883-2940. 
Takada, H., Onda, T., Ogura, N., 1990. Determination of Polycyclic Aromatic-Hydrocarbons in Urban 650 Street Dusts and Their Source Materials by Capillary Gas-Chromatography. Environ Sci Technol 24, 651 1179-1186.

652 Trac, L.N., Schmidt, S.N., Holmstrup, M., Mayer, P., 2019. Headspace Passive Dosing of Volatile 653 Hydrophobic Organic Chemicals from a Lipid Donor-Linking Their Toxicity to Well-Defined Exposure 654 for an Improved Risk Assessment. Environ Sci Technol 53, 13468-13476.

655 Trac, L.N., Schmidt, S.N., Mayer, P., 2018. Headspace passive dosing of volatile hydrophobic 656 chemicals - Aquatic toxicity testing exactly at the saturation level. Chemosphere 211, 694-700. US EPA, 2016. Estimation Programs Interface Suite ${ }^{\mathrm{TM}}$ for Microsoft ${ }^{\circledR}$ Windows, v 4.1. United States Environmental Protection Agency, Washington, DC, USA.

659 Veith, G.D., Call, D.J., Brooke, L.T., 1983. Structure toxicity relationships for the fathead minnow, 660 Pimephales-Promelas - narcotic industrial-chemicals. Can J Fish Aquat Sci 40, 743-748.

661 Vergauwen, L., Schmidt, S.N., Stinckens, E., Maho, W., Blust, R., Mayer, P., Covaci, A., Knapen, D., 662 2015. A high throughput passive dosing format for the fish embryo acute toxicity test. Chemosphere 663 139, 9-17.

664 Yalkowsky, S.H., Orr, R.J., Valvani, S.C., 1979. Solubility and Partitioning .3. Solubility of Halobenzenes 665 in Water. Ind Eng Chem Fund 18, 351-353. 
Toxicity of dodecylbenzene to algae, crustacean, and fish - Passive dosing of highly hydrophobic liquids at the solubility limit

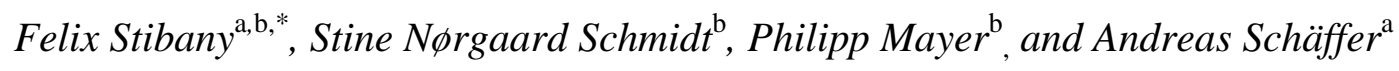

${ }^{a}$ Institute for Environmental Research (Biology V), RWTH Aachen University, Worringerweg 1, 52074 Aachen, Germany

${ }^{\mathrm{b}}$ Department of Environmental Engineering, Technical University of Denmark, Bygningstorvet B115, 2800 Kgs. Lyngby, Denmark

* Corresponding author: felix.stibany@rwth-aachen.de

Current affiliation for Stine Nørgaard Schmidt: National Research Council, U.S. Environmental Protection Agency, Office of Research and Development, Center for Environmental Measurement and Modeling, Atlantic Coastal Environmental Sciences Division, Narragansett, Rhode Island, USA

\section{Highlights}

1) Aquatic toxicity testing of a highly hydrophobic liquid at its solubility limit

2) Constant exposure by the application of passive dosing

3) Suggested improvements of current standard test systems for hydrophobic chemicals

4) Full base-set of ecotoxicological data according to REACH (three trophic levels)

5) Evidence against the existence of a generic $\log K_{O w}$ cut-off in baseline toxicity 


\section{Declaration of interests}

$\bigotimes$ The authors declare that they have no known competing financial interests or personal relationships that could have appeared to influence the work reported in this paper.

$\square$ The authors declare the following financial interests/personal relationships which may be considered as potential competing interests: 